\author{
Grzegorz Domański, Bogumił Konarzewski, \\ Zdzisław Pawłowski, Krzysztof Zaremba, Janusz Marzec, \\ Artur Trybuła, Robert Kurjata
}

\title{
A Simple Method of Determining the Effective Attenuation Coefficient
}

\author{
Institute of Radioelectronics, Warsaw University of Technology, \\ Nowowiejska 15/19, 00-665 Warsaw, Poland \\ e-mail: g.domanski@ire.pw.edu.pl
}

This paper presents a simple method of determining the effective attenuation coefficient from steady-state diffuse reflectance measurements.

Key words: optical parameters, effective attenuation coefficient, reflectance measurements.

\section{Introduction}

Visible and near-infrared (NIR) light interacts with biological tissues by absorption and scattering $[1,2,3,4,5]$. It is important to know the optical properties of tissues in order to properly choose the measurement geometry in optical tomography [2, 3]. Also, optical properties themselves can potentially provide information about the tissue's metabolic status or diagnose a disease [2,3]. Optical properties of phantom tissues and biological tissues have been described by authors using various methods and techniques $[6,7]$. However, it is still a problem of how to determine these optical properties quickly and relatively cheaply [6, 7]. The aim of this work was to develop a simple and cheap method for effective in vivo estimation of the attenuation coefficient. 


\section{Theory and Modelling}

In this section a simple model of radiation transport is described, which was used to derive an effective absorption coefficient from spatially resolved measurements of diffuse reflectance. A diffusion model was used to generate analytical expressions for the reflectance. For an infinitive medium the solution of the diffusion equation is given by [3]:

$$
\Phi(r)=\frac{I_{0}}{4 \pi D} \cdot \frac{\exp \left(-\mu_{\text {eff }} r\right)}{r}
$$

where: $\Phi$ is the photon fluence rate $\left[\mathrm{W} / \mathrm{cm}^{2}\right], I_{0}$ is the source intensity $[\mathrm{W}], D$ is the diffusion coefficient [6] given by $D=\frac{1}{3\left(\mu_{a}+\mu_{s}^{\prime}\right)}, \mu_{\mathrm{a}}$ is an absorption coefficient $\left[\mathrm{cm}^{-1}\right]$ and $\mu_{\mathrm{s}}$ is the scattering coefficient $\left[\mathrm{cm}^{-1}\right], \mu_{s}^{\prime}=(1-g) \mu_{s}$ being the reduced scattering coefficient, $g$ is the anisotropy coefficient, and $\mu_{e f f}=\sqrt{\frac{\mu_{a}}{D}}$ is the effective attenuation coefficient [7]. From equation (1) it can clearly be seen that in the diffusion theory for an infinitive medium light attenuation in tissue depends only on $\mu_{\text {eff }}$ rather than on the absorption coefficient or the scattering coefficient alone. The knowledge of $\mu_{\text {eff }}$ enables us to quickly evaluate light attenuation between a source and a detector.

A quotient of two photon fluence rates for two different source-to-detector distances $r$ and $r_{0}$ is given by

$$
\frac{\Phi}{\Phi_{0}}=\frac{r_{0}}{r} \exp \left(-\mu_{\text {eff }}\left(r-r_{0}\right)\right)
$$

Its natural logarithm is

$$
\ln \left(\frac{\Phi}{\Phi_{0}}\right)=\ln \left(\frac{r_{0}}{r}\right)-\mu_{\text {eff }}\left(r-r_{0}\right)
$$

For large source-to-detector distances, the above function behaves asymptotically as a linear function with a slope $-\mu_{\text {eff }}$, which makes its determination fast and easy. 
Let the logarithm of the measured quotient of light intensity be denoted by $y=\ln \left(\frac{\Phi}{\Phi_{0}}\right)$ and the distances from the source to the detector at the measured points by $r_{i}$, where $i=$ $1, \ldots, n$. The values $y$, measured at points $r_{i}$, are denoted by $y_{i}$. Then

$$
f\left(\mu_{\text {eff }}, r\right)=\ln \left(\frac{\Phi}{\Phi_{0}}\right)=\ln \left(\frac{r_{0}}{r}\right)-\mu_{\text {eff }}\left(r-r_{0}\right)
$$

In order to determine the value of $\mu_{\text {eff }}$, the sum of squares of the deviations of the theoretical signal values $y=f\left(\mu_{e f f} r\right)$ from the measured signals $y_{i}$ is minimized. Let the sum be written as

$$
S=\sum_{i=1}^{n}\left(y_{i}-f\left(\mu_{e f f}, r_{i}\right)\right)^{2}
$$

Minimization of $S$ in view of $\mu_{\text {eff }}$ leads to

$$
\frac{d S}{d \mu_{\text {eff }}}=0
$$

By computing the derivative we obtain

$$
\begin{aligned}
\frac{\partial S}{\partial \mu_{\text {eff }}} & =\sum_{i=1}^{n} \frac{\partial f}{\partial \mu_{\text {eff }}}\left[\left(y_{i}-f\left(\mu_{\text {eff }}, \mathrm{r}_{i}\right)\right)^{2}\right]= \\
& =\sum_{i=1}^{n} 2\left(y_{i}-f\left(\mu_{\text {eff }}, \mathrm{r}_{i}\right)\right)\left[-\frac{\partial f}{\partial \mu_{\text {eff }}}\left(\mu_{\text {eff }}, \mathrm{r}_{i}\right)\right]= \\
& =2 \sum_{i=1}^{n}\left(y_{i}-f\left(\mu_{\text {eff }}, \mathrm{r}_{i}\right)\right)\left(r_{i}-r_{0}\right)
\end{aligned}
$$

After comparing it to zero, we obtain

$$
2 \sum_{i=1}^{n}\left(y_{i}-\ln \left(\frac{\mathrm{r}_{0}}{\mathrm{r}_{i}}\right)+\mu_{\text {eff }}\left(\mathrm{r}_{i}-\mathrm{r}_{0}\right)\right)\left(\mathrm{r}_{i}-\mathrm{r}_{0}\right)=0
$$

From the above we finally have

$$
\sum_{i=1}^{n}\left(y_{i}-\ln \left(\frac{\mathrm{r}_{0}}{\mathrm{r}_{i}}\right)\right)\left(\mathrm{r}_{i}-\mathrm{r}_{0}\right)+\mu_{\text {eff }} \sum_{\mathrm{i}=1}^{\mathrm{n}}\left(\mathrm{r}_{i}-\mathrm{r}_{0}\right)^{2}=0
$$


The value of $\mu_{\text {eff }}$, which minimizes the cost function $S$, can be computed from the following formula

$$
\mu_{\text {eff }}=-\frac{\sum_{i=1}^{n}\left(y_{i}-\ln \left(\frac{\mathrm{r}_{0}}{\mathrm{r}_{i}}\right)\right)\left(\mathrm{r}_{i}-\mathrm{r}_{0}\right)}{\sum_{\mathrm{i}=1}^{\mathrm{n}}\left(\mathrm{r}_{i}-\mathrm{r}_{0}\right)^{2}}
$$

With the aim of finding the uncertainty of the estimated parameter $\mu_{\text {eff }}$, let us assume that the $y_{i}$ measurement is a random variable with the variance $\operatorname{var}\left(y_{i}\right)$, and the random variables $y_{i}$ are independent for different values of $i$. The variance $v \operatorname{ar}\left(\mu_{\mathrm{eff}}\right)$ is then given by

$$
\operatorname{var}\left(\mu_{\text {eff }}\right)=\sum_{i=1}^{n}\left(\frac{\partial \mu_{\text {eff }}}{\partial y_{i}}\right)^{2} \operatorname{var}\left(\mathrm{y}_{i}\right)
$$

Its derivative is given by

$$
\frac{\partial \mu_{\text {eff }}}{\partial y_{i}}=-\frac{r_{i}-r_{0}}{\sum_{k=1}^{n}\left(r_{k}-r_{0}\right)^{2}}
$$

The variance $\operatorname{var}\left(\mu_{\mathrm{eff}}\right)$ is

$$
\operatorname{var}\left(\mu_{\text {eff }}\right)=\frac{\sum_{i=1}^{n}\left(r_{i}-r_{0}\right)^{2} \operatorname{var}\left(y_{i}\right)}{\left(\sum_{i=1}^{n}\left(r_{i}-r_{0}\right)^{2}\right)^{2}}
$$

The standard deviation $\sigma\left(\mu_{\text {eff }}\right)$ is

$$
\sigma\left(\mu_{\text {eff }}\right)=\sqrt{\operatorname{var}\left(\mu_{\text {eff }}\right)}=\frac{\sqrt{\sum_{i=1}^{n}\left(r_{i}-r_{0}\right)^{2} \operatorname{var}\left(y_{i}\right)}}{\sum_{i=1}^{n}\left(r_{i}-r_{0}\right)^{2}}
$$


The variance $\operatorname{var}\left(y_{i}\right)$ is expressed by the measured light intensity

$$
\begin{aligned}
\operatorname{var}\left(y_{i}\right)=\operatorname{var}\left[\ln \left(\frac{\Phi_{i}}{\Phi_{0}}\right)\right] & =\left[\frac{\partial}{\partial \Phi_{i}}\left(\ln \left(\frac{\Phi_{i}}{\Phi_{0}}\right)\right)\right]^{2} \operatorname{var}\left(\Phi_{i}\right)= \\
& =\left(\frac{\Phi_{0}}{\Phi_{i}} \frac{1}{\Phi_{0}}\right)^{2} \operatorname{var}\left(\Phi_{i}\right)=\frac{\operatorname{var}\left(\Phi_{i}\right)}{\Phi_{i}^{2}}
\end{aligned}
$$

If we assume that the main source of the uncertainty of the measured photon fluence rate $\Phi$ is random optical contact of the detector with the tissue, then the standard deviation of the measured value of $\Phi$ is proportional to $\Phi$, with the coefficient of proportionality of $\alpha$

$$
\sigma(\Phi)=\alpha \Phi
$$

The variance $\operatorname{var}\left(y_{i}\right)$ is expressed by the variance of the measured photon fluence rate

$$
\operatorname{var}\left(y_{i}\right)=\frac{\operatorname{var}\left(\Phi_{i}\right)}{\Phi_{\imath}^{2}}=\alpha^{2}
$$

The variance of the estimated effective attenuation coefficient $v a r\left(\mu_{\text {eff }}\right)$ is

$$
\operatorname{var}\left(\mu_{\text {eff }}\right)=\frac{\sum_{i=1}^{n}\left(r_{i}-r_{0}\right)^{2} \alpha^{2}}{\left(\sum_{i=1}^{n}\left(r_{i}-r_{0}\right)^{2}\right)^{2}}=\frac{\alpha^{2}}{\sum_{i=1}^{n}\left(r_{i}-r_{0}\right)^{2}}
$$

The standard deviation of $\mu_{\text {eff }}$ is then given by

$$
\sigma\left(\mu_{\text {eff }}\right)=\frac{\alpha}{\sqrt{\sum_{i=1}^{n}\left(r_{i}-r_{0}\right)^{2}}}
$$

For a semi-infinitive medium the solution of the diffusion equation is given by

$$
\Phi(r, z)=\frac{I_{0}}{4 \pi D} \cdot\left[\frac{\exp \left(-\mu_{\text {eff }} \sqrt{\left(z-z_{0}\right)^{2}+r^{2}}\right)}{\sqrt{\left(z-z_{0}\right)^{2}+r^{2}}}-\frac{\exp \left(-\mu_{\text {eff }} \sqrt{\left(z+z_{0}+2 z_{b}\right)^{2}+r^{2}}\right)}{\sqrt{\left(z+z_{0}+2 z_{b}\right)^{2}+r^{2}}}\right]
$$


where $z_{0}=\frac{1}{\mu_{s}^{\prime}}, z_{b}$ is the distance to the extrapolated boundary, $z_{b}=2 D \frac{1+R_{\text {eff }}}{1-R_{\text {eff }}}$, $R_{\text {eff }}=-1.440 n_{\text {rel }}^{-2}+0.710 n_{\text {rel }}^{-1}+0.668+0.0636 n_{\text {rel }}, n_{\text {rel }}=\frac{n_{\text {tissue }}}{n_{\text {air }}}$ is the relative refraction index of the tissue compared to that of air.

For this model the estimated value of parameter $x=\mu_{\text {eff }}$ is found by minimizing the cost function $E(x)$

$$
E(x)=\sum_{i=1}^{n}\left(f\left(r_{i}, x\right)-y_{i}\right)^{2}
$$

where

$$
f\left(r_{i}, x\right)=\ln \left(\frac{\Phi\left(r_{i}, x\right)}{\Phi\left(r_{0}, x\right)}\right)
$$

The condition for the minimum is $\frac{d E}{d x}=0$.

Then, the value of the derivative is given by

$$
\begin{gathered}
\frac{d E}{d x}=\sum_{i=1}^{n} 2\left(f\left(r_{i}, x\right)-y_{i}\right) \frac{\partial f}{\partial x} \\
\frac{\partial f}{\partial x}=\frac{\Phi\left(r_{0}, x\right)}{\Phi\left(r_{i}, x\right)} \frac{1}{\Phi^{2}\left(r_{0}, x\right)}\left(\frac{\partial \Phi\left(r_{i}, x\right)}{\partial x} \Phi\left(r_{0}, x\right)-\frac{\partial \Phi\left(r_{0}, x\right)}{\partial x} \Phi\left(r_{i}, x\right)\right) \\
\frac{\partial f}{\partial x}=\frac{1}{\Phi\left(r_{i}, x\right) \Phi\left(r_{0}, x\right)}\left(\frac{\partial \Phi\left(r_{i}, x\right)}{\partial x} \Phi\left(r_{0}, x\right)-\frac{\partial \Phi\left(r_{0}, x\right)}{\partial x} \Phi\left(r_{i}, x\right)\right) \\
\frac{\partial f}{\partial x}=\frac{\partial \Phi\left(r_{i}, x\right)}{\partial x} \frac{1}{\Phi\left(r_{i}, x\right)}-\frac{\partial \Phi\left(r_{0}, x\right)}{\partial x} \frac{1}{\Phi\left(r_{0}, x\right)}
\end{gathered}
$$

The value of the second derivative is given by

$$
\frac{d^{2} E}{d x^{2}}=\sum_{i=1}^{n} 2\left[\left(\frac{\partial f}{\partial x}\right)^{2}+\left(f\left(r_{i}, x\right)-y_{i}\right) \frac{\partial^{2} f}{\partial x^{2}}\right]
$$


The approximate value of $x=\mu_{\text {eff }}$ obtained from (8) can be improved by iteration

$$
x^{\prime}=x-\frac{\left(\frac{d E}{d x}\right)}{\left(\frac{d^{2} E}{d x^{2}}\right)}
$$

Let replace function $\Phi$ from (17) by solving the diffusion equation for a semi-infinitive medium, written as

$$
\Phi(r, x)=\frac{I_{1}}{4 \pi D} \cdot\left[\frac{\exp \left(-x R_{1}\right)}{R_{1}}-\frac{\exp \left(-x R_{2}\right)}{R_{2}}\right]
$$

where $R_{1}=\sqrt{\left(z-z_{0}\right)^{2}+r^{2}}, R_{2}=\sqrt{\left(z+z_{0}+2 z_{b}\right)^{2}+r^{2}}$.

If $R_{10}=R_{1}\left(r_{0}\right), R_{1 i}=R\left(r_{i}\right), R_{20}=R_{2}\left(r_{0}\right), R_{2 i}=R_{2}\left(r_{i}\right)$, function $f$ can be written as

$$
f\left(r_{i}, z\right)=\ln \left[\frac{\frac{\exp \left(-x R_{1 i}\right)}{R_{1 i}}-\frac{\exp \left(-x R_{2 i}\right)}{R_{2 i}}}{\frac{\exp \left(-x R_{10}\right)}{R_{10}}-\frac{\exp \left(-x R_{20}\right)}{R_{20}}}\right]
$$

If

$$
\begin{gathered}
\varphi_{i}=\frac{\exp \left(-x R_{1 i}\right)}{R_{1 i}}-\frac{\exp \left(-x R_{2 i}\right)}{R_{2 i}} \\
\varphi_{0}=\frac{\exp \left(-x R_{10}\right)}{R_{10}}-\frac{\exp \left(-x R_{20}\right)}{R_{20}}
\end{gathered}
$$

then

$$
f=\ln \left(\frac{\varphi_{i}}{\varphi_{0}}\right)
$$




$$
\begin{aligned}
& \frac{\partial f}{\partial x}=\frac{\varphi_{0}}{\varphi_{i}} \frac{\frac{\partial \varphi_{i}}{\partial x} \varphi_{0}-\frac{\partial \varphi_{0}}{\partial x} \varphi_{i}}{\varphi_{0}^{2}}=\frac{\partial \varphi_{i}}{\partial x} \frac{1}{\varphi_{i}}-\frac{\partial \varphi_{0}}{\partial x} \frac{1}{\varphi_{0}} \\
& \frac{\partial \varphi_{i}}{\partial x}=-\exp \left(-x R_{1 i}\right)+\exp \left(-x R_{2 i}\right) \\
& \frac{\partial \varphi_{0}}{\partial x}=-\exp \left(-x R_{10}\right)+\exp \left(-x R_{20}\right) \\
& \frac{\partial^{2} f}{\partial x^{2}}=\left[\frac{\partial^{2} \varphi_{i}}{\partial x^{2}} \frac{1}{\varphi_{i}}+\frac{\partial \varphi_{i}}{\partial x}\left(-\frac{1}{\varphi_{i}^{2}} \frac{\partial \varphi_{i}}{\partial x}\right)\right]-\left[\frac{\partial^{2} \varphi_{0}}{\partial x^{2}} \frac{1}{\varphi_{0}}+\frac{\partial \varphi_{0}}{\partial x}\left(-\frac{1}{\varphi_{0}^{2}} \frac{\partial \varphi_{0}}{\partial x}\right)\right] \\
& \frac{\partial^{2} f}{\partial x^{2}}=\frac{1}{\varphi_{i}}\left[\frac{\partial^{2} \varphi_{i}}{\partial x^{2}}-\frac{1}{\varphi_{i}}\left(\frac{\partial \varphi_{i}}{\partial x}\right)^{2}\right]-\frac{1}{\varphi_{0}}\left[\frac{\partial^{2} \varphi_{0}}{\partial x^{2}}-\frac{1}{\varphi_{0}}-\left(\frac{\partial \varphi_{0}}{\partial x}\right)^{2}\right] \\
& \frac{\partial^{2} \varphi_{i}}{\partial x^{2}}=R_{1 i} \exp \left(-x R_{1 i}\right)-R_{2 i} \exp \left(-x R_{2 i}\right) \\
& \frac{\partial^{2} \varphi_{i}}{\partial x^{2}}=R_{10} \exp \left(-x R_{10}\right)-R_{20} \exp \left(-x R_{20}\right)
\end{aligned}
$$

The variance of $x$ can be computed from

$$
\begin{gathered}
\operatorname{var}(x)=\sum_{i=1}^{n}\left|\frac{\partial}{\partial y_{i}} \frac{\frac{d E}{d x}}{\frac{d^{2} E}{d x^{2}}}\right|^{2} \sigma^{2}\left(y_{i}\right) \\
\frac{\partial}{\partial y_{i}} \frac{\frac{d E}{d x}}{\frac{d^{2} E}{d x^{2}}}=\frac{1}{\left(\frac{d^{2} E}{d x^{2}}\right)^{2}}\left[\frac{\partial}{\partial y_{i}}\left(\frac{d E}{d x}\right) \frac{d^{2} E}{d x^{2}}-\frac{\partial}{\partial y_{i}}\left(\frac{d^{2} E}{d x^{2}}\right) \frac{d E}{d x}\right]
\end{gathered}
$$




$$
\begin{gathered}
\frac{\partial}{\partial y_{i}} \frac{d E}{d x}=-2 \frac{d f}{d x} \\
\frac{\partial}{\partial y_{i}} \frac{d^{2} E}{d x^{2}}=-2 \frac{d^{2} f}{d x^{2}} \\
\operatorname{var}(x)=\frac{4 \alpha^{2}}{\left(\frac{d^{2} E}{d x^{2}}\right)^{4}} \sum_{i=1}^{n}\left|\frac{\partial f}{\partial x} \frac{d^{2} E}{d x^{2}}-\frac{d E}{d x} \frac{d^{2} f}{d x^{2}}\right|^{2}
\end{gathered}
$$

The above equations serve as a background theory for estimating the measuring error.

\section{Materials and Methods}

The surface light intensity distributions were measured in vivo for human forearms by means of a one-channel microprocessor system. The system consists of a light detector (silicon photodiode), a switched integrator and a microprocessor with a built-in 10-bit analogue-to-digital converter. The tissue under examination was coupled to a light detector by fiber optics. The device was controlled from a personal computer via a serial interface RS232. The light source was a LED-emitting light of the wavelength of about $660 \mathrm{~nm}$. The block schematic of the microprocessor system for the measurements of optical parameters is shown in Figure 1.

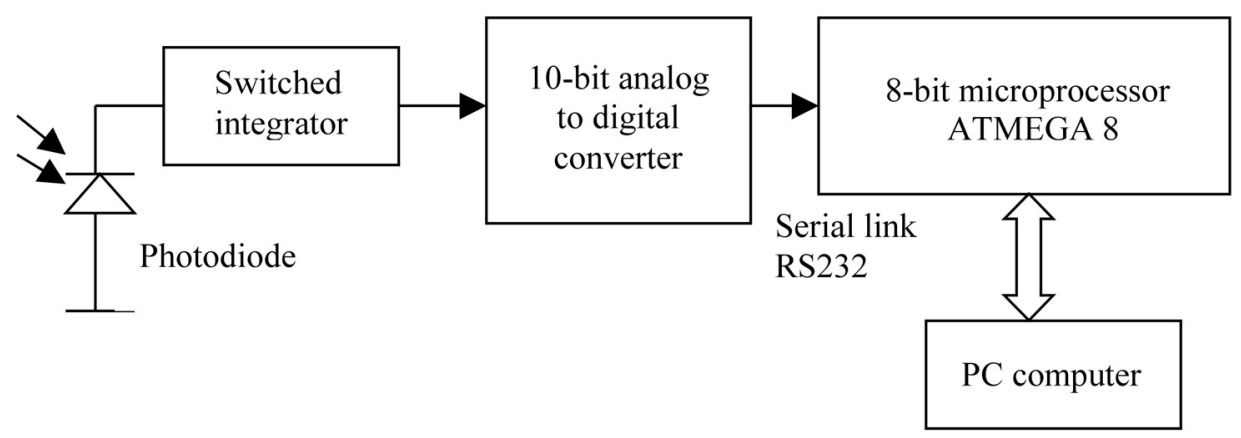

Figure 1. A block schematic of the microprocessor system for measurements of optical parameters. 


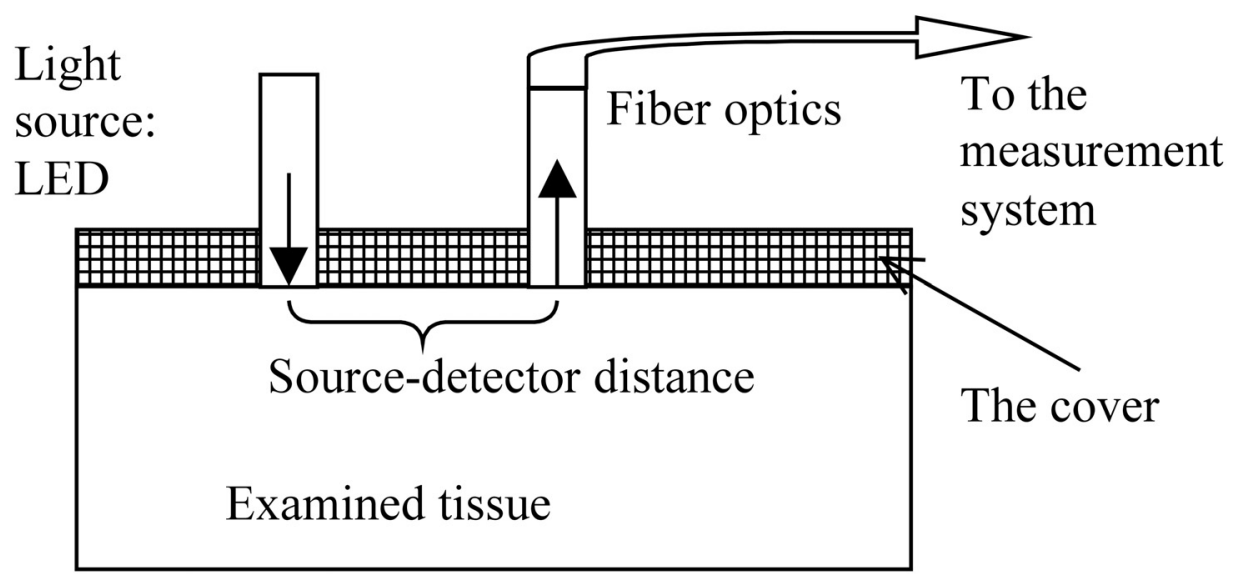

Figure 2. Fixing the source on the tissue.

Table 1. The results of $\mu_{\text {eff }}$ coefficient in vivo determination for human forearms.

\begin{tabular}{|c|c|c|}
\hline Person & Forearm & $\mu_{\text {eff }}\left[\mathrm{cm}^{-1}\right]$ \\
\hline \hline A & Left & $2.03 \pm 0.19$ \\
\hline A & Right & $2.20 \pm 0.19$ \\
\hline B & Left & $2.52 \pm 0.19$ \\
\hline B & Right & $2.40 \pm 0.19$ \\
\hline C & Left & $2.25 \pm 0.19$ \\
\hline C & Right & $2.16 \pm 0.19$ \\
\hline D & Left & $2.59 \pm 0.18$ \\
\hline D & Right & $2.37 \pm 0.19$ \\
\hline E & Right & $2.05 \pm 0.19$ \\
\hline
\end{tabular}


Figure 2 shows how the source was placed on the tissue examined. Both the source and the detector were placed on top of the forearm. The plastic cover served as a positioning support.

\section{Results}

The optical parameter $\mu_{\text {eff }}$ was measured in vivo on human forearms by means of our method. The persons studied were 25-56 year-old males. The surface light intensity distributions were measured by means of our system, and then the $\mu_{\text {eff }}$ coefficient estimations were computed using the theory described in (8). These values were used as starting values for an iteration procedure given in (23). The final $\mu_{\text {eff }}$ coefficient results for human forearms in vivo are shown in Table 1.

The range of the estimated values of the effective attenuation coefficient was 2-3 $\mathrm{cm}^{-1}$. These results agree with those reported in literature [6, 7]. However, our values are probably slightly overestimated due to the inaccuracies in the applied model of light transport in tissue, given by equation (17). Using a more accurate model of light propagation, for example the Monte Carlo simulation, better values of the effective attenuation coefficient could be obtained.

\section{Discussion and Conclusions}

Our method is simple, fast and inexpensive. It allows finding the effective attenuation coefficient from light intensity measurements for at least two places on the tissue examined. A measurement theory was developed and tested with real data. The estimated values of the optical parameters can be used as starting values for an iteration procedure in the Monte Carlo simulation procedure.

\section{References}

[1] Arridge SR, Hebden JC. Optical imaging in Medicine: II. Modelling and reconstruction. Physics in Medicine and Biology 1997; 42: 841-853.

[2] Frostig RD (editor). In Vivo Optical Imaging of Brain Function, CRC Press, Boca Raton, FL, 2002. 
[3] Tuchin VV (editor). Handbook of Optical Biomedical Diagnostics, SPIE Press, Bellingham, 2002.

[4] Vo-Dinh T. Biomedical Photonics Handbook, CRC Press, Boca Raton, Fl, 2003.

[5] Milstein AB, Oh S, Reynolds JS, Webb KJ, Bouman CA, Millane RP. Three-dimensional optical diffusion tomography with experimental data. Optics Letters 2002; 27(2) 95-97.

[6] Kienle A, Glanzamann T. In vivo determination of the optical properties of muscle with time-resolved reflectance using a layered model. Phys Med Biol 1999; 44: 2689-2702.

[7] Marquez G, Wang LV. White light oblique incidence reflectometer for measuring absorption and reduced scattering spectra of tissue-like turbid media. Optics Express 1997; 1: 454-460. 